\title{
Use of tazarotene foam for the treatment of acne vulgaris
}

This article was published in the following Dove Press journal:

Clinical, Cosmetic and Investigational Dermatology

27 May 2014

Number of times this article has been viewed

\section{Stamatis Gregoriou \\ Eleftheria Kritsotaki \\ Alexandros Katoulis \\ Dimitris Rigopoulos}

Second Department of Dermatology and Venereology, Attikon Hospital, University of Athens Medical School, Athens, Greece
Correspondence: Dimitris Rigopoulos Second Department of Dermatology and Venereology, Attikon Hospital, University of Athens Medical School, I Rimini Street, Athens 12462, Greece Tel +302105832396

$\mathrm{Fax}+302105832397$

Email stamgreg@yahoo.gr
Abstract: Acne vulgaris is a common inflammatory chronic disease of the pilosebaceous unit. It often requires long-term treatment, resulting in increased demand for topical medications that are popular with patients in order to achieve long-term compliance. Tazarotene foam $0.1 \%$ is a novel formulation of tazarotene. We review efficacy and tolerability studies of the new formulation, and suggest a possible place for the product in the management of acne vulgaris.

Keywords: retinoids, efficacy, safety, tolerability

\section{Introduction to the management of acne vulgaris}

Acne vulgaris is a common inflammatory chronic disease of the pilosebaceous unit. It is considered to be a multifactorial condition that results from four primary pathophysiologic processes:

- abnormal follicular keratinization that leads to ductal obstruction

- increased and altered sebum production that is androgen-induced

- follicular colonization and proliferation of Propionibacterium acnes

- altered adaptive immune response and inflammation. ${ }^{1}$

Even though it is often perceived as a self-limited and not physically disabling disease of adolescence, its prevalence remains high into adulthood, and its psychological impact can be striking, contributing to lower self-esteem, anxiety, and depression. ${ }^{2}$ Consequently, there is significant patient-driven demand for effective acne therapies, including prescribed medications and over-the-counter products. In addition, taking into account the need for long-term treatment, there is increased need for topical medications that are popular with patients in order to achieve long-term compliance. As a result, agents are available in a variety of formulations. These include topical antibiotics, retinoids, and benzoyl peroxide in monotherapy or in combination products. Systemic medications also include antibiotics and retinoids, as well as hormonal agents.

\section{Pharmacology, mode of action, and pharmacokinetics of tazarotene foam}

Tazarotene is a member of the acetylenic class of retinoids. Chemically, tazarotene is ethyl 6-([4,4-dimethylthiochroman-6-yl]ethynyl)nicotinate. Tazarotene is a retinoid prodrug that is converted to its active form, the cognate carboxylic acid of tazarotene, by rapid desertification in animals and man. Tazarotenic acid binds to all three members of the retinoic acid receptor (RAR) family - RAR $\alpha$, RAR $\beta$, and RAR $\gamma$ - but shows relative selectivity for RAR $\beta$ and $R A R \gamma$ and may modify gene expression. 
Tazarotene is approved for the treatment of psoriasis and acne. Only the $0.1 \%$ strength is approved by the US Food and Drug Administration (FDA) for the treatment of acne. Cream $0.1 \%$ is indicated for acne vulgaris, and gel $0.1 \%$ is indicated for mild-to-moderate acne vulgaris, whereas foam $0.1 \%$ is indicated for moderate-to-severe acne. Comparison studies between tazarotene, adapalene, and tretinoin report mixed results, with some researchers suggesting comparable efficacy and tolerability, while others report greater efficacy and irritation with tazarotene..$^{3-6}$

The basis of the therapeutic effect of tazarotene in acne may be due to its antihyperproliferative, normalizing-ofdifferentiation, and anti-inflammatory effects. The cellular mechanisms and inflammatory cascades that might correlate with acne pathogenesis and are modulated by topical tazarotene include:

- reduced expression of hyperproliferative keratins K6 and $\mathrm{K} 16$, which are increased during comedogenesis

- suppression of activation of the activator protein 1 , which results in reduced expression of several matrix metalloproteinases from keratinocytes, which have been shown to be increased in acne vulgaris

- decreased expression of Toll-like receptor (TLR) 2 and decrease in ligand binding with $P$. acnes, which results in inhibition of the TLR-2-induced innate response that triggers inflammation in acne

- increased epidermal turnover, with reduction in postinflammatory hyperpigmentation

- normalization of epidermal cellular differentiation and decreased hyperkeratinization

- downregulated expression of the epidermal growth factor receptor. $^{7}$

Tazarotene foam $0.1 \%$ was FDA-approved in 2012 for the treatment of acne vulgaris in patients 12 years old and over. It is the first retinoid in a foam preparation. The new foam vehicle is emulsion-based, ethanol-free, and moisturizing. It contains $1 \mathrm{mg} / \mathrm{g}$ tazarotene in an aqueous-based foam vehicle consisting of butylated hydroxytoluene, ceteareth-12, citric acid anhydrous, diisopropyl adipate, light mineral oil, potassium citrate monohydrate, potassium sorbate, purified water, and sorbic acid. ${ }^{8}$

Tazarotene is a retinoid prodrug that is converted in the skin and plasma to its biologically active form of tazarotenic acid by de-esterification. Tazarotene and tazarotenic acid are extensively bound (more than 99\%) to human plasma proteins. Little parent compound can be detected in the plasma. Secondary metabolites of tazarotenic acid (the sulfoxide, the sulfone, and an oxygenated derivative of tazarotenic acid) are eliminated through urinary and fecal pathways.

A Phase I study was conducted to assess the relative bioavailability of active metabolite tazarotenic acid after topical application of two different formulations of tazarotene - foam or gel. Twenty-nine patients with moderate-to-severe acne vulgaris received a mean once-daily dose for 22 days of $3.7 \mathrm{~g}$ tazarotene foam $0.1 \%$ or gel $0.1 \%$ in a ratio of $13: 16$, applied to approximately $15 \%$ of the body surface area (face, chest, upper back, and shoulders). Systemic exposure to tazarotene and tazarotenic acid was measured through maximum plasma concentration $\left(\mathrm{C}_{\max }\right)$, time to $\mathrm{C}_{\max }$, through concentration in plasma, elimination half-life, and areas under the plasma concentration-time curve from time zero to time of last measurable concentration and during a dosage interval (AUCs). Statistical analysis of these pharmacokinetic parameters for tazarotene and its active metabolite showed significantly higher $\mathrm{AUC}_{0-\mathrm{t}}$ and $\mathrm{C}_{\max }$ with tazarotene gel than with tazarotene foam by an average of approximately 1.8 - to 2.2 -fold. The results of this trial indicated that tazarotene foam $0.1 \%$ offers less tazarotenic acid systemic exposure and favorable safety for the treatment of acne vulgaris when compared to tazarotene gel $0.1 \%{ }^{9}$

\section{Efficacy studies}

Two Phase III multicenter, randomized, double-blind, vehiclecontrolled, parallel-group studies were conducted in participants with acne vulgaris to assess the efficacy of tazarotene foam $0.1 \%$ compared with vehicle foam. These studies were carried out in 39 centers over Canada and the US. ${ }^{10}$

The first study involved 744 participants and the second 742 aged $12-45$ years with moderate-to-severe acne vulgaris according to the Investigator Static Global Assessment (ISGA) scale, with 25-50 facial inflammatory lesions, and 30-125 facial noninflammatory lesions at baseline. The primary end points of these Phase III studies were:

- lesion reduction in two of three lesion counts (inflammatory, noninflammatory, and total) from baseline to week 12 (end of treatment)

- percentage of subjects who had a score of 0-1 (clear or almost-clear skin) on the ISGA scale

- a minimum 2-grade improvement at week 12.

Patients were randomized to receive treatment with either tazarotene foam $0.1 \%$ or vehicle foam once daily for 12 weeks. Results from both clinical trials were favorable for the treatment arm that received tazarotene foam. Reduction in lesion counts at week 12 was statistically greater $(P<0.001)$ for the tazarotene foam compared with 
the vehicle foam. An ISGA score of 0 or 1 was achieved by a higher percentage of participants on tazarotene foam than participants on vehicle foam. In both studies, a significantly greater $(P<0.001)$ proportion of patients on the tazarotene foam arm achieved at least a 2-grade improvement on the ISGA scale by the end of treatment compared with the control arm. Reductions in lesion counts and improvement in ISGA scale at week 12 are presented in Table $1 .^{10}$

\section{Safety and tolerability issues}

Safety data from the Phase III studies are presented in Table 2. Most adverse reactions were mild to moderate in severity. Severe adverse reactions occurred in $3.0 \%$ of subjects; $2.6 \%$ of patients discontinued due to local skin reactions. ${ }^{10}$

Two single-center, evaluator-blinded, randomized, vehiclecontrolled, Phase I patch studies were performed to evaluate tazarotene foam $0.1 \%$ for cumulative irritation potential and contact-sensitization potential. Thirty-nine patients were enrolled in the first study, and 254 patients in the second. ${ }^{11}$

In the 21-day cumulative irritation trial, $200 \mathrm{~mL}$ of each study product - tazarotene foam $0.1 \%$, sodium lauryl sulfate $0.5 \%$ (positive control), distilled water (negative control), and vehicle foam - was applied to participants' backs once daily for $24 \pm 1$ hours, and the application site was evaluated for signs of irritation. Results of this study showed that tazarotene foam can potentially induce irritation. Mean converted cumulative irritation score statistically differed among study products $(P<0.0001)$, and in pairwise comparisons was statistically higher with tazarotene foam than the positive control $(P<0.0001)$, the negative control $(P<0.0001)$, and vehicle foam $(P<0.0001)$. No serious adverse events $(\mathrm{AEs})$ were reported.

In the contact-sensitization study, patches of either tazarotene $0.1 \%$ or vehicle foam were applied on participants' backs for a 3-week induction phase (three times per week for $48 \pm 2$ or $72 \pm 2$ hours), followed by a 1-week challenge and a 2 -week rechallenge phase (single 48-hour application) at the investigators' discretion with interim 2-week rest periods. Patch sites were visually assessed and scored according to grading scales. Three participants demonstrated questionable sensitization reactions and underwent a rechallenge; none of the participants displayed conclusive contact sensitization. Three application-site AEs were considered to be productrelated; none of the AEs led to study discontinuation. Results of this second study indicated a low potential for contactsensitization reactions for both tazarotene foam and vehicle foam. Overall, these studies demonstrated an acceptable tolerability and safety profile for tazarotene foam $0.1 \%$.

Tazarotene foam $0.1 \%$ was evaluated for phototoxic and photoallergic potential in two single-center, evaluationblinded, randomized, vehicle-controlled Phase I studies conducted in $2012 .{ }^{12}$ In the phototoxic potential study, 38 participants were enrolled and exposed to study products tazarotene foam $0.1 \%$ and vehicle foam patches - and no foam patches (blank) over 24 hours. One set each was exposed to ultraviolet (UV) irradiation, UV and visible (VIS) light, and no irradiation. Inflammatory skin responses and superficial effects were evaluated. Overall, $89 \%$ of the participants had no phototoxic reaction, and $19.4 \%$ had an unclear reaction. Phototoxic reaction or photoirritation was limited to only three participants $(8.3 \%)$. No AEs were reported.

In the photoallergic potential study, 59 subjects were enrolled and exposed to study products for a 3-week induction phase (six consecutive 24-hour applications, two patches per set, tazarotene/foam patches), followed by a 1-week challenge and a 1-week rechallenge phase (single 24-hour application, three patches per set, tazarotene/ foam/blank patches) with interim 2-week rest periods.

Table I Reductions in lesion counts and improvement in Investigator Global Assessment at week 12

\begin{tabular}{|c|c|c|c|c|}
\hline & $\begin{array}{c}\text { Tazarotene } \\
\text { foam, } n=37 \text { I }\end{array}$ & $\begin{array}{l}\text { Vehicle } \\
\text { foam, } n=372\end{array}$ & $\begin{array}{c}\text { Tazarotene } \\
\text { foam, } n=373\end{array}$ & $\begin{array}{l}\text { Vehicle } \\
\text { foam, } n=369\end{array}$ \\
\hline \multicolumn{5}{|l|}{ Inflammatory lesions } \\
\hline Mean absolute reduction from baseline & 18.0 & 14.0 & 18.0 & 15.0 \\
\hline Mean percentage reduction from baseline & $58 \%$ & $45 \%$ & $55 \%$ & $45 \%$ \\
\hline \multicolumn{5}{|l|}{ Noninflammatory lesions } \\
\hline Mean absolute reduction from baseline & 28.0 & 17.0 & 26.0 & 18.0 \\
\hline Mean percentage reduction from baseline & $55 \%$ & $33 \%$ & $57 \%$ & $41 \%$ \\
\hline \multicolumn{5}{|l|}{ Total lesions } \\
\hline Mean absolute reduction from baseline & 46.0 & 31.0 & 43.0 & 33.0 \\
\hline Mean percentage reduction from baseline & $56 \%$ & $39 \%$ & $56 \%$ & $43 \%$ \\
\hline \multicolumn{5}{|l|}{ IGA, n (\%) } \\
\hline Minimum 2-grade improvement and IGA of 0 or I & 107 (29\%) & 60 (16\%) & 103 (28\%) & 49 (13\%) \\
\hline
\end{tabular}

Abbreviation: IGA, Investigator Global Assessment. 
Table 2 Incidence of adverse reactions at the application site in $1 \%$ of patients treated with tazarotene foam $0.1 \%$

\begin{tabular}{lll}
\hline $\begin{array}{l}\text { Adverse reactions at } \\
\text { the application site }\end{array}$ & $\begin{array}{l}\text { Tazarotene } \\
\text { foam, } \mathbf{n = 7 4 4}\end{array}$ & $\begin{array}{l}\text { Vehicle } \\
\text { foam, } \mathbf{n = 7 4 I}\end{array}$ \\
\hline $\begin{array}{l}\text { Patients with any } \\
\text { adverse reaction, } \mathrm{n}(\%)\end{array}$ & $163(22)$ & $19(3)$ \\
$\begin{array}{l}\text { Irritation } \\
\text { Dryness }\end{array}$ & $107(14)$ & $9(\mathrm{I})$ \\
Erythema & $50(7)$ & $8(\mathrm{I})$ \\
Exfoliation & $48(6)$ & $3(<\mathrm{I})$ \\
Pain & $44(6)$ & $3(<\mathrm{I})$ \\
Photosensitivity & $9(1)$ & 0 \\
(including sunburn) & $8(1)$ & $3(<\mathrm{I})$ \\
Pruritus & $7(1)$ & $3(<\mathrm{I})$ \\
Dermatitis & $6(1)$ & $\mathrm{I}(<\mathrm{I})$ \\
\hline
\end{tabular}

Application sites were exposed to UVB irradiation and VIS light after each application during the induction phase. After 10-17 days, participants received both UVA and UVA/UVB irradiation, UVA/UVB plus VIS irradiation, or no irradiation during the challenge phase. Among the participants that were administered tazarotene foam $0.1 \%$, a rate of $50 \%$ after irradiation and $60 \%$ after no irradiation showed no visible reactions at 72 hours. Grade 3 erythema was limited to $4 \%$ of the participants. Seven AEs were reported, but none was considered to be related to the study product. Results from these studies suggest that tazarotene foam $0.1 \%$ shows minor photoirritant potential and low potential for phototoxic or photoallergic reactions.

Like all retinoids, tazarotene foam $0.1 \%$ is pregnancy category $\mathrm{X}$. Both clinical and in vitro findings have revealed that vitamin $\mathrm{A}$ and its active derivatives (retinoids) exert a wide variety of effects on vertebrate embryonic body-shaping and organogenesis, tissue homeostasis, cell proliferation, differentiation, and apoptosis. ${ }^{13}$ Retinoic acid can induce teratogenesis of the fetus of many animals, including humans, induced mainly by RAR and RAR ligands. The ligands of the retinoid $\mathrm{X}$ receptor cannot induce teratogenesis, but they can enhance the teratogenesis of the RAR stimulus. RARs can also affect the development of the fetus by adjusting the expression of the other genes. ${ }^{14}$

It is not known what level of exposure to tazarotene is required for teratogenicity in humans. ${ }^{15}$ There were five pregnancies in women who participated in the tazarotene-foam studies. Only one subject was exposed to the active drug, and her exposure was for 25 days. She delivered a healthy baby. In clinical trials with the other topical tazarotene formulations, nine pregnant women were exposed to the active drug. One woman elected to terminate her pregnancy, and the other eight women delivered healthy babies. ${ }^{8}$
According to the package insert, topical tazarotene should be avoided in women of childbearing potential who are contemplating pregnancy or who are unwilling to take adequate precautions to avoid pregnancy. It is recommended that a negative serum or urine pregnancy test be obtained prior to beginning therapy.

\section{Effects on adherence and quality of life}

A major consideration in prescribing topical retinoids is patient tolerability and adherence. An advisory panel exploring optimization of the use of tazarotene in clinical practice in order to minimize the irritation of previously available formulations recommended that the choice of concentration should be based on such factors as the irritability of the patient's skin and the thickness of plaques in psoriasis. Irritation should be managed by reducing the concentration or frequency of application, or by adding a topical corticosteroid to therapy. ${ }^{16}$ Short-contact therapy has also been explored with the previously available $0.1 \%$ gel formulation, with improved results. Patients with plaque psoriasis applying the $0.1 \%$ gel formulation for 20 minutes, followed by washing with water, developed much less frequent and severe irritant contact dermatitis than traditional treatment with the same formulation. ${ }^{17}$

Results of the cumulative irritation study showed that tazarotene foam can potentially induce irritation. ${ }^{11}$ Results from a clinical study showed that $14 \%$ of the patients using tazarotene foam reported irritation, while only $1 \%$ of the patients using the vehicle foam experienced such an effect (Table 2).${ }^{18}$ Patients who did not complete the clinical study numbered 66 among those using tazarotene foam and 39 among those using the vehicle foam (Table 3). ${ }^{18}$ Among those stating

Table 3 Analysis of patients lost to follow-up

\begin{tabular}{lll}
\hline & $\begin{array}{l}\text { Tazarotene } \\
\text { foam }\end{array}$ & $\begin{array}{l}\text { Vehicle } \\
\text { foam }\end{array}$ \\
\hline Started & 372 & 372 \\
Completed & 306 & 333 \\
Not completed & 66 & 39 \\
$\quad$ Adverse event & $\mathrm{II}$ & $\mathrm{I}$ \\
Lost to follow-up & 14 & 14 \\
Noncompliance with study product & $\mathrm{I}$ & $\mathrm{I}$ \\
Withdrawal by subject & 32 & 16 \\
Relocation & 0 & 4 \\
Pregnancy & 2 & $\mathrm{I}$ \\
Protocol violation & 2 & 0 \\
Took excluded medication & 2 & 0 \\
Did not meet eligibility criteria & $\mathrm{I}$ & 2 \\
Change in work situation & $\mathrm{I}$ & 0 \\
\hline
\end{tabular}


Table 4 Impact on quality of life of patients applying tazarotene foam or vehicle in the 12-week studies using the Dermatology Life Quality Index (DLQI) and Children's Dermatology Life Quality Index (CDLQI)

\begin{tabular}{|c|c|c|}
\hline & $\begin{array}{l}\text { Tazarotene } \\
\text { foam }\end{array}$ & $\begin{array}{l}\text { Vehicle } \\
\text { foam }\end{array}$ \\
\hline \multicolumn{3}{|l|}{ DLQI } \\
\hline Number of participants analyzed & $17 \mid$ & 165 \\
\hline \multicolumn{3}{|c|}{ Change in DLQI score from baseline at weeks $2,4,8$, and I 2 in participants } \\
\hline \multicolumn{3}{|c|}{17 years of age or older, mean \pm standard deviation } \\
\hline Week 2, n=171,165 & $-0.1 \pm 4.98$ & $-2.1 \pm 3.68$ \\
\hline Week 4, n=166,167 & $-1.9 \pm 4.60$ & $-2.5 \pm 3.85$ \\
\hline Week $8, n=160,155$ & $-2.9 \pm 4.86$ & $-2.7 \pm 4.40$ \\
\hline Week I2, n=154, 155 & $-3.6 \pm 5.22$ & $-3.1 \pm 4.31$ \\
\hline \multicolumn{3}{|l|}{ CDLQI } \\
\hline Number of participants analyzed & 162 & 179 \\
\hline \multicolumn{3}{|c|}{ Change of CDLQI from baseline at weeks 2, 4, 8, and I 2 in participants } \\
\hline \multicolumn{3}{|c|}{16 years old or younger, mean \pm standard deviation } \\
\hline Week 2, n=162, 179 & $1.1 \pm 4.16$ & $-1.4 \pm 3.21$ \\
\hline Week 4, n=153, 180 & $-0.6 \pm 3.45$ & $-1.4 \pm 3.23$ \\
\hline Week 8, n=|48,|7| & $-1.2 \pm 3.53$ & $-1.9 \pm 3.11$ \\
\hline Week $12, n=146,169$ & $-1.7 \pm 4.09$ & $-2.0 \pm 3.46$ \\
\hline
\end{tabular}

an adverse event as the reason for not completing the study, eleven were in the tazarotene-foam group, while only one was in the vehicle group. In addition, in the category of withdrawal by subject, 32 patients belonged in the tazarotene group and 16 in the vehicle group. Consequently, one has to assume that decreased adherence is to be expected with tazarotene foam when compared to products with no active ingredients. Comparison studies evaluating adherence with patients using other topical retinoid products should provide more valuable information on the subject.

When considering the effects of treatment of tazarotene foam on quality of life using the Dermatology Life Quality Index on patients older than 17 years and Children's Dermatology Life Quality Index on patients younger than 17 years, no information on statistical analysis is provided. ${ }^{18}$ However, the results indicate an early burden on the quality of life of patients using the tazarotene foam for the first 4 weeks, which could be attributed to possible irritation by tazarotene. Results indicate a greater improvement in quality of life of patients using tazarotene when compared to those using the vehicle at week 12, which could be attributed to the efficacy of tazarotene foam in acne vulgaris (Table 4). ${ }^{18}$

\section{Place in therapy and conclusion}

Guidelines can never encompass every clinical situation. However, recommendations based on review of the literature improve the quality of acne therapy in general. Personal experiences should always be critically evaluated, and therapeutic decision making should take into account the consented therapeutic recommendations as well as the type of acne and the severity of the disease. The European evidenced-based guidelines for the treatment of acne were made with the goals of promoting adherence of patients, and reducing serious conditions and scarring, as well as antibiotic resistance. ${ }^{19}$ They include a medium-strength recommendation for topical retinoid monotherapy for patients with comedonal acne. The German Society of Dermatology and the German Association of Dermatologists also suggest retinoid monotherapy as the treatment of choice for comedonal acne. ${ }^{20}$ An international committee of physicians and researchers in the field of acne concluded that topical retinoids should be the foundation of treatment for most patients with acne, because retinoids target the microcomedo, the precursor to all acne lesions, and they also have intrinsic anti-inflammatory effects, thus targeting two pathogenic factors in acne. ${ }^{21}$ The American Academy of Dermatologists treatment guidelines for acne suggest a level A strength of recommendation for topical retinoids based on level I evidence from literature available at the time of publication..$^{22}$ Consequently, tazarotene should be considered as the treatment of choice for comedonal acne, and one should expect an additional effect on inflammatory acne lesions, particularly after prolonged use.

There is no undisputed conclusion about the efficacy and tolerability of tazarotene when compared to adapalene and tretinoin. Consequently, the needs of the individual patient should be considered when deciding which topical retinoid to prescribe. Even though the foam is a new vehicle with excellent moisturizing properties, no studies exist to compare efficacy and tolerability when compared with the gel- and cream-tazarotene formulations. When selecting to prescribe the tazarotene-foam formulation, physicians should inform the patient about the possibility of irritation, particularly during the first 2 weeks of application, and explain that this is associated with the product's mode of action and will eventually contribute to good therapeutic results. Additional hydrating products to lessen the irritation, as well as shortcontact application schedules, might be employed during the first few days to allow the patient to become gradually accustomed to the tazarotene effects.

Combination therapy with retinoid/antimicrobial products has been proposed as the treatment of choice in patients presenting with comedonal and papulopustular acne. ${ }^{22}$ Even though no studies exist on the efficacy of tazarotene foam employed in conjunction with an antimicrobial agent, such as benzoyl peroxide or clindamycin, common sense indicates that such a combination therapy might have merit. Issues that 
might be of concern include the possibility of additional irritation caused by benzoyl peroxide and the impact the use of two products might have on patient adherence.

In conclusion, tazarotene foam $0.1 \%$ has been shown to have an excellent efficacy and good tolerability profile in the studies published. Experience accumulated through prescription, as well as comparison studies with other topical retinoids and the other tazarotene formulations, will provide substantial information about its possible advantages and disadvantages in the future.

\section{Disclosure}

The authors report no conflicts of interest in this work.

\section{References}

1. Dawson AL, Dellavalle RP. Acne vulgaris. BMJ. 2013;346:f2634.

2. Tasoula E, Gregoriou S, Chalikias J, et al. The impact of acne vulgaris on quality of life and psychic health in young adolescents in Greece. Results of a population survey. An Bras Dermatol. 2012;87: 862-869.

3. Pariser D, Colón LE, Johnson LA, Gottschalk RW. Adapalene $0.1 \%$ gel compared to tazarotene $0.1 \%$ cream in the treatment of acne vulgaris. J Drugs Dermatol. 2008;7:s18-s23.

4. Thiboutot D, Arsonnaud S, Soto P. Efficacy and tolerability of adapalene $0.3 \%$ gel compared to tazarotene $0.1 \%$ gel in the treatment of acne vulgaris. J Drugs Dermatol. 2008;7:s3-s10.

5. Webster GF, Berson D, Stein LF, Fivenson DP, Tanghetti EA, Ling M. Efficacy and tolerability of once-daily tazarotene $0.1 \%$ gel versus once-daily tretinoin $0.025 \%$ gel in the treatment of facial acne vulgaris: a randomized trial. Cutis. 2001;67:4-9.

6. Leyden JJ, Tanghetti EA, Miller B, Ung M, Berson D, Lee J. Once-daily tazarotene $0.1 \%$ gel versus once-daily tretinoin $0.1 \%$ microsponge gel for the treatment of facial acne vulgaris: a double-blind randomized trial. Cutis. 2002;69:12-19.

7. Del Rosso JQ, Tanghetti E. A status report on topical tazarotene in the management of acne vulgaris. J Drugs Dermatol. 2013;12:s53-s58.
8. Epstein EL, Stein Gold L. Safety and efficacy of tazarotene foam for the treatment of acne vulgaris. Clin Cosmet Investig Dermatol. 2013;6:123-125.

9. Jarratt M, Werner CP, Alió Saenz AB. Tazarotene foam versus tazarotene gel: a randomized relative bioavailability study in acne vulgaris. Clin Drug Investig. 2013;33:283-289.

10. Feldman SR, Werner CP, Alió Saenz AB. The efficacy and tolerability of tazarotene foam, $0.1 \%$, in the treatment of acne vulgaris in 2 multicenter, randomized, vehicle-controlled, double-blind studies. $J$ Drugs Dermatol. 2013;12:438-446.

11. Berg JE, Bowman JP, Saenz AB. Cumulative irritation potential and contact sensitization potential of tazarotene foam $0.1 \%$ in 2 phase 1 patch studies. Cutis. 2012;90:206-211.

12. Hogan DJ, Saenz AB. Phototoxic and photoallergic potential of tazarotene foam $0.1 \%$ in 2 phase 1 patch studies. Cutis. 2012;90:266-271.

13. Mark M, Ghyselinck NB, Chambon P. Function of retinoid nuclear receptors: lessons from genetic and pharmacological dissections of the retinoic acid signaling pathway during mouse embryogenesis. Аппи Rev Pharmacol Toxicol. 2006;46:451-480.

14. Tzimas G, Nau H. The role of metabolism and toxicokinetics in retinoid teratogenesis. Curr Pharm Des. 2001;7:803-831.

15. Phillips TJ. An update on the safety and efficacy of topical retinoids. Cutis. 2005;75:14-22.

16. Roeder A, Schaller M, Schäfer-Korting M, Korting H.C. Tazarotene: therapeutic strategies in the treatment of psoriasis, acne and photoaging. Skin Pharmacol Physiol. 2004;17:111-118.

17. Veraldi S, Caputo R, Pacifico A, Peris K, Soda R, Chimenti S. Short contact therapy with tazarotene in psoriasis vulgaris. Dermatology. 2006;212:235-237.

18. Stiefel. A study to evaluate the safety and efficacy of tazarotene foam, $0.1 \%$, in subjects with common facial acne-W0260-301. Available from: http://clinicaltrials.gov/ct2/show/results/NCT01017146?sect=Xf015. NLM identifier: NCT01017146. Accessed December 30, 2013.

19. Nast A, Dréno B, Bettoli V, et al. European evidence-based (S3) guidelines for the treatment of acne. J Eur Acad Dermatol Venereol. 2012;26 Suppl 1:1-29.

20. Nast A, Bayerl C, Borelli C, et al. [S2k-guideline for therapy of acne]. J Dtsch Dermatol Ges. 2010;8 Suppl 2:s1-s59.

21. Zaenglein AL, Thiboutot DM. Expert committee recommendations for acne management. Pediatrics. 2006;118:1188-1199.

22. Strauss JS, Krowchuk DP, Leyden JJ, et al. Guidelines of care for acne vulgaris management. JAm Acad Dermatol. 2007;56:651-663.
Clinical, Cosmetic and Investigational Dermatology

\section{Publish your work in this journal}

Clinical, Cosmetic and Investigational Dermatology is an international, peer-reviewed, open access, online journal that focuses on the latest clinical and experimental research in all aspects of skin disease and cosmetic interventions. All areas of dermatology will be covered; contributions will be welcomed from all clinicians and

\section{Dovepress}

basic science researchers globally. This journal is indexed on CAS The manuscript management system is completely online and includes a very quick and fair peer-review system, which is all easy to use. Visit http://www.dovepress.com/testimonials.php to read real quotes from published authors. 